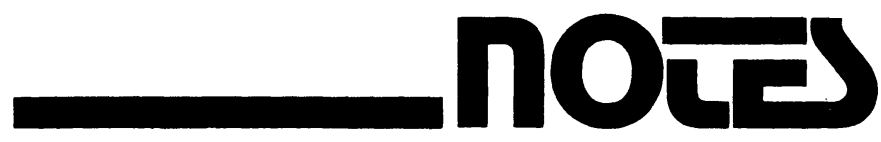

\title{
Planning for Interruptions
}

\author{
JoAnne Simpson Growney \\ Bloomsburg State College \\ Bloomsburg, PA 17815
}

Organizing is what you do before you do something,
so that when you do it, it's not all mixed up.
-Christopher Robin in A. A. Milne's Winnie the Pooh

Nothing is particularly hard if you divide it into small jobs.

- Henry Ford

It is Tuesday morning. Faculty members Jones and Smith are each in their offices preparing for eleven o'clock classes.

Professor Jones has left his door open. His students who pass by the office stop in with questions and comments. Professor Jones interrupts his lecture preparation to respond to their queries. These students leave the office pleased and satisfied with the help provided by their instructor. But eleven o'clock arrives without Jones' class lecture being fully prepared. His presentation is disorganized, and both he and the class members are dissatisfied with the results.

The door to Professor Smith's office is, on the other hand, closed and locked. Students with questions pass by the door and find their instructor unavailable. They leave dissatisfied. When eleven o'clock arrives, Professor Smith has completed her class preparation. The class material is well organized and she presents it clearly. Both she and her students are pleased and satisfied with the learning that took place in their hour together. Nevertheless, later Professor Smith is dismayed when she learns of student dissatisfaction with her prior unavailability to answer individual questions.

The choices faced by Professors Jones and Smith-either to isolate themselves and concentrate on a task that needs to be thought through carefully without interruption or to permit interruptions that may prevent completion of the desired task-provide a dilemma for many individuals. Teachers, students, managers, parents - to name a few-all face this conflict repeatedly. On the one hand are unpredictable events to which attention should be given as the need arises. On the other hand are tasks that require uninterrupted periods of thought.

The purpose of this article is to develop a mathematical model that confirms the wisdom of planning activities so as to minimize the detrimental effect of interruptions.

\section{The parable of the watchmakers}

The value of planning for interruptions is well illustrated by the following tale [4]:

Once upon a time there were two watchmakers, Hora and Tempus. Each produced very fine watches which began to be in great demand. Their workshop phones rang frequently, bringing orders from new customers. Hora prospered but Tempus become poorer and poorer and finally lost his shop. What was the secret of Hora's success?

Both men made watches that consisted of about 1000 parts. Tempus had constructed his so 
that if several pieces were assembled and he had to put the assembly down-for example, to answer the phone-all the pieces fell apart and had to be reassembled from scratch. Naturally, the better his customers liked his work, the more they told their friends-who interrupted his work to place orders for more watches. As a result it became more and more difficult for Tempus to find enough uninterrupted time to finish a watch.

Hora, on the other hand, had designed his watches so that he could put together components of about ten parts. As with Tempus, if he was interrupted while working on a particular assembly, the pieces fell apart and had to be reassembled from scratch. But the ten-part components, once completed, were "stable" and could be set aside for later use as a unit. Ten of the components could be assembled into a larger stable subsystem. Later the assembly of the ten larger subsystems constituted the whole watch.

The obvious secret of Hora's success was the use of "stable" subsystems that allowed him to pay only a small penalty in watch-assembly time for each interruption. What the parable fails to tell us, however, is the price that Hora paid to develop his method. That is, we do not know how many hours or days or weeks it took Hora to organize his system of assembly. We see only his success without any history of the size of his initial investment.

In general, individuals are often unwilling to invest time to organize lengthy tasks into "stable" subtasks because the time required to get organized is an initial cost, often great, that appears to lengthen even more the time that the task will require. However, our mathematical model for analysis of interruptions will reveal that extra organizational time is often a wise investment.

For Tempus and Hora, for example, if $p=0.01$ is the probability of interruption during the addition of any given part to the watch assembly, the formula given below for $T$ can be used to estimate that the time required for Tempus to complete a watch is almost 2000 times as great as that for Hora. Thus, even if organizing his watchmaking into stable subsystems was a very lengthy process for Hora, it proved an essential ingredient in his success and provided substantial improvement over the assembly method of Tempus.

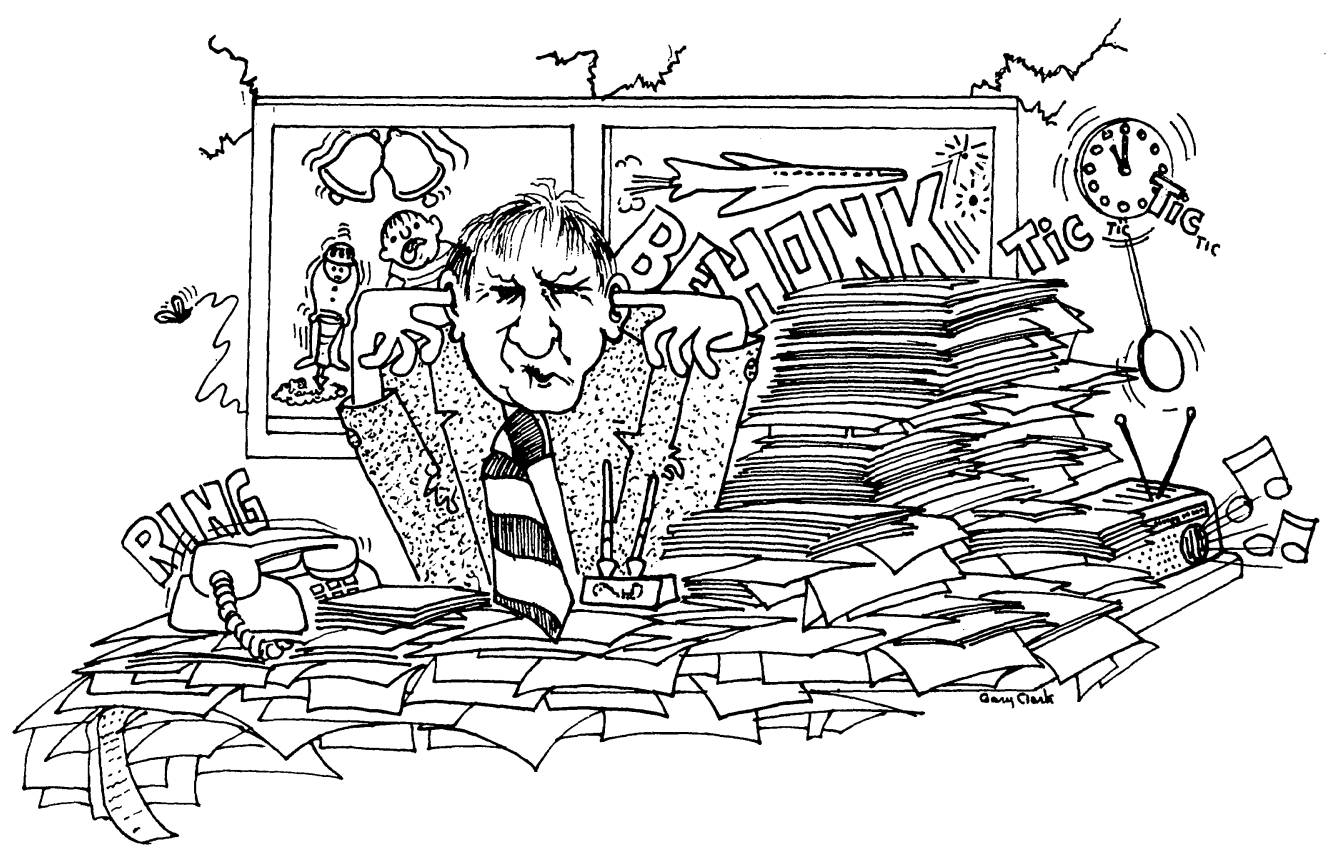




\section{A model for estimating the effect of interruptions}

Suppose a certain project is to be performed and that the project is divided into $s$ stable subtasks, each of which requires $U$ consecutive uninterrupted time units for its completion. (For example, in The Parable of the Watchmakers, Tempus' method for constructing a watch has $s=1$ and $U=1000$; Hora's method has $s=111$ and $U=10$.) If no interruptions occur, the total time required for completion of the desired project is $s \cdot U$.

Further, suppose that interruptions occur randomly with probability $p$ during any given time unit. Our model will assume two basic properties concerning the nature of the interruptions. The first is that any interruption has no effect on the pattern of subsequent interruptions - that is, interruptions are independent. The second assumption is that each interruption during a stable subtask requires starting over at the beginning of that subtask-that is, interruptions are devastating.

The assumptions that interruptions are independent and devastating are not wholly realistic. Yet in cases in which interruptions are troublesome, these are not unreasonable suppositions. For, in such cases, it seems that no matter how many interruptions we deal with we cannot change the likelihood of more later-hence the reasonable assumption of independence. Furthermore, the frustration associated with being diverted from a task that requires sustained concentration makes a later return to the task almost like starting over. Hence the assumption that interruptions are devastating also is reasonable.

If $T$ denotes the expected number of time units required for completion of the desired project, then

$$
T=\frac{s}{p}\left[\frac{1}{(1-p)^{U}}-1\right]
$$

where $s, p$, and $U$ are as defined above.

By application of l'Hôpital's rule, we can determine that

$$
\lim _{p \rightarrow 0} T=\lim _{p \rightarrow 0}\left\{\frac{s\left[\frac{1}{(1-p)^{U}}-1\right]}{p}\right\}=s \cdot U .
$$

This limiting value, $s \cdot U$, is the total time required for completion of the project if no interruptions occur.

We now show how formula (1) for $T$ can be deduced. For background details from the theory of probability, consult references [1], [2], or [3].

The probability that an interruption will not occur during a given time interval is $(1-p)$. The probability of no interruption during $U$ consecutive time intervals is $(1-p)^{U}$-since the interruptions are independent. The quantity $(1-p)^{U}$ may also be interpreted as the proportion of starts that are completed. Its reciprocal $1 /(1-p)^{U}$ thus gives the expected number of starts per completed subtask. We then have that the expected number of subtask starts required for completion of the entire project is

$$
\left.\begin{array}{l}
\text { Expected number of } \\
\text { subtask starts required } \\
\text { for project completion }
\end{array}\right\}=\frac{1}{(1-p)^{U}} \cdot s .
$$

Since the probability of no interruptions during $U$ consecutive time intervals is $(1-p)^{U}$, the probability that an interruption will occur during one of $U$ consecutive time intervals is $\left[1-(1-p)^{U}\right]$. This probability may also be interpreted as the proportion of starts that are interrupted-equivalently, as the expected number of interruptions per subtask start. Combining this with (2) gives 
$\left.\begin{array}{l}\text { Expected number of } \\ \text { interruptions during } \\ \text { project completion }\end{array}\right\}=\left[1-(1-p)^{U}\right] \cdot \frac{1}{(1-p)^{U}} \cdot s$.

To obtain the equation for $T$ it remains only to multiply equation (3) by the expected length of time-on-task that elapses between interruptions- that is, the expected number of time units spent per interruption-which is equal to $1 / p$. Thus we have

$$
\left.\begin{array}{l}
\begin{array}{l}
\text { Expected number of } \\
\text { time units required } \\
\text { for project completion }
\end{array}
\end{array}\right\}=\frac{1}{p} \cdot\left[1-(1-p)^{U}\right] \cdot \frac{1}{(1-p)^{U}} \cdot s
$$

which simplifies to yield formula (1).

Returning to the watchmaker parable, we have

For Tempus:

$$
\begin{aligned}
s & =1 \\
U & =1000 \\
p & =0.01 \\
T & =\frac{1}{.01}\left[\frac{1}{(1-.01)^{1000}}-1\right] \approx 2,316,000 \text { time units. }
\end{aligned}
$$

For Hora:

$$
\begin{aligned}
s & =111 \\
U & =10 \\
p & =0.01 \\
T & =\frac{1}{.01}\left[\frac{1}{(1-.01)^{10}}-1\right] \approx 1174 \text { time units. }
\end{aligned}
$$

Hora, in producing a watch that requires assembly of 1110 parts (including the various stable subsystems), loses an average of 64 time units because of starting over after interruptions. For Tempus, the time loss is so great that he can reasonably expect never to complete a watch under the given circumstances.

Our interruption model, based on the watchmaker parable, has the following moral:

Time invested in organizing a complex project into stable subtasks is amply returned through a reduction in the time lost because of interruptions.

A simple example will illustrate the moral.

EXAMPLE. Two political science students, Jim and Jean, have each been assigned to write a brief paper analyzing and comparing different voting methods. Each estimates that the rough draft will take one hour of intense concentration - that is, it must be done without interruptions. Any interruption will be so devastating that starting over will be required. For both Jim and Jean interruptions occur randomly but, on the average, every twenty minutes.

(a) Jim started writing his rough draft at 6 P.M. last evening. Under the given conditions, what is the expected time at which he is finished?

Analysis. Using the notation of the interruption model, we have

$$
\begin{aligned}
s & =1 \text { (stable subtasks), } \\
U & =60 \text { (consecutive uninterrupted minutes), }
\end{aligned}
$$




$$
p=\frac{1}{20}=0.05 \text { (likelihood of interruption }
$$

Substitution of these values into the formula for $T$ yields

$$
T=\frac{1}{.05}\left[\frac{1}{(1-.05)^{60}}-1\right] \approx 414 \text { minutes. }
$$

Thus Jim's rough draft required an estimated seven hours of "time-on-task" and, if his interruptions diverted him for only brief periods, his completion time would be expected to be around 1 A.M. this morning.

(b) Jean was astounded when a sleepy-eyed Jim told her how long it had taken him to complete his rough draft. Based on his experience, she has decided to try a different approach. Jean believes that with fifteen minutes of intense concentration she can outline her paper and divide it into four sections that will each take fifteen minutes to write. If she is interrupted during any fifteen-minute interval, she will need to start over and spend fifteen more minutes on that portion of her task. However, once the outline or any of the subsections is complete, no interruption will affect it.

Jean thus has five fifteen-minute tasks to complete without interruption. How long will it take her? If she begins at 6 P.M. tonight, when can she expect to finish?

Analysis. Using the notation of the interruption model, we have

$$
\begin{aligned}
& s=5 \text { (stable subtasks), } \\
& U=15 \text { (consecutive uninterrupted minutes), } \\
& p=\frac{1}{20}=0.05 \text { (likelihood of interruption } \\
& \text { during any given minute). }
\end{aligned}
$$

Substitution of these values into the formula for $T$ yields

$$
T=\frac{1}{.05}\left[\frac{1}{(1-.05)^{15}}-1\right] \approx 116 \text { minutes. }
$$

Thus Jean's rough draft will require about two hours for completion. If she starts at 6 P.M. this evening she can expect to complete her task at about 7:56 P.M. $+I$-where $I$ is the number of minutes actually devoted to her interruptions.

\section{When is organizational time worth the investment?}

In the example above, Jean's investment of fifteen extra minutes to organize her paper resulted in an expected savings-when compared with Jim's situation-of almost five hours. We easily conclude that her initial investment was worthwhile. However, a general question emerges: Under what conditions is it likely to be worthwhile to invest time to organize a lengthy task into stable subtasks to reduce the startover time required by interruptions?

A partial answer results from consideration of a special case. Suppose the probability $p$ of interruption during any given time unit and the number $U$ of consecutive uninterrupted time units required to complete the desired task are related by

$$
U=\frac{1}{p} \text {. }
$$

In this situation, with the task not divided into more than one subtask, we have

$$
T=U \cdot\left[\frac{1}{(1-1 / U)^{U}}-1\right] \text {. }
$$




\section{JUST FOR FUN}

Paul has recently become aware of the evidences of his own aging and has determined to reverse the process and become physically fit. Being a rational fellow he has consulted his physician before embarking on a fitness program. The doctor has recommended for Paul a daily stint of eight uninterrupted minutes on a treadmill. If Paul is interrupted during his effort then he must start over and complete eight more minutes without interruption.

Sounds simple! However, Paul has another affliction. He also suffers from a chronic skin disorder. Randomly, but on the average of once every two minutes, Paul is interrupted with the necessity to stop and scratch an itch.

How does Paul fare as he tries to carry out his physical fitness program? The truth may never be known but you can simulate Paul's frustrating experience in the following manner:

Repeatedly toss a coin, letting each toss represent a minute for Paul on the treadmill. Let each toss that results in a head designate a minute in which Paul must stop to scratch. A tail permits him to keep jogging. How many tosses altogether are required to simulate a string of eight consecutive uninterrupted minutes of jogging?

From this we obtain

$$
\frac{T}{U}=\left[\left(\frac{U}{U-1}\right)^{U}-1\right]
$$

TABLE 1 lists a sample of values of $U$ and corresponding values for $T / U$. More generally, we observe that $T / U$ is a decreasing function of $U$ and, as $U$ becomes large, $T / U$ approaches $(e-1)$. (L'Hôpital's rule is useful in evaluating this limit; for illustration of the method see, for example, [5].)

\begin{tabular}{|c|c|c|c|c|c|c|c|c|c|c|}
\hline$U$ & 2 & 3 & 4 & 5 & 7 & 10 & 15 & 25 & 50 & 100 \\
\hline$T / U$ & 3 & 2.375 & 2.160 & 2.052 & 1.942 & 1.868 & 1.815 & 1.775 & 1.746 & 1.732 \\
\hline
\end{tabular}

TABLE 1

Thus, in the case in which $U=1 / p$, the "time-on-task" will be lengthened by over $70 \%$ because of interruptions. In such case, an initial investment of organizational time-to divide the task into stable subtasks - is well worth considering. In general, if we value our time, we should never attempt to complete an uninterruptable task unless the time it requires is substantially less than the length of time we expect between interruptions.

The question of how to minimize the sum of organizational time plus time wasted due to interruptions is difficult to solve in general. Nevertheless, an example will illustrate the tractability of the problem in any specific case.

Consider a situation in which a project to be completed requires 100 time units. Suppose that the task is subdivided into $s$ uninterruptable subtasks each requiring $U$ uninterrupted time units so that we have $s U=100$. Suppose further that the probability of interruption during any given time unit is $p=0.05$. TABLE 2 gives the values of $T$ for various subdivisions of the task into $s$ stable subtasks. In this case we have

$$
T=20 s \cdot\left[\frac{1}{(.95)^{100 / s}}-1\right]
$$

\begin{tabular}{|l|c|c|c|c|c|c|c|c|}
\hline$s$ & 1 & 2 & 5 & 10 & 20 & 25 & 50 & 100 \\
\hline$T$ & 3360 & 480 & 179 & 134 & 117 & 114 & 108 & 105 \\
\hline
\end{tabular}

TABLE 2 
Of course, the time wasted because of interruptions decreases as $s$ increases. But to assume that a large number of subtasks is optimal is naive; it ignores the time required for organizing the project into stable subtasks.

For the project under consideration, let us suppose that the organizational time required is proportional to the number of additional subtasks that must be devised. That is, suppose there is a positive constant $C$ for which $C \cdot(s-1)$ gives the cost in organizational time. TABLE 3 lists values of $C \cdot(s-1)$ for the values $C=0.1,1,10$, and for the $s$-values of TABLE 2. (We still assume that $s \cdot U=100$.)

\begin{tabular}{|r|l|l|l|l|l|l|l|l|}
\hline$C$ & 1 & 2 & 5 & 10 & 20 & 25 & 50 & 100 \\
\hline 0.1 & 0 & .1 & .4 & .9 & 1.9 & 2.4 & 4.9 & 9.9 \\
1 & 0 & 1 & 4 & 9 & 19 & 24 & 49 & 99 \\
10 & 0 & 10 & 40 & 90 & 190 & 240 & 490 & 990 \\
\hline
\end{tabular}

TABLE 3. Values of $C \cdot(s-1)$.

Minimizing the time required for the project requires finding that value of $s$ for which $T+C \cdot(s-1)$ is least. Circled values in TABLE 4 show these minima.

\begin{tabular}{|c|c|c|c|c|c|c|c|c|}
\hline$C$ & 1 & 2 & 5 & 10 & 20 & 25 & 50 & 100 \\
\hline 0.1 & 3360 & 480.1 & 179.4 & 134.9 & 118.9 & 116.4 & (12.9 & 114.9 \\
\hline 1 & 3360 & 481 & 183 & 143 & 136 & 138 & 157 & 204 \\
\hline 10 & 3360 & 490 & 219 & 224 & 307 & 354 & 598 & 1095 \\
\hline
\end{tabular}

TABLE 4. Values of $\boldsymbol{T}+\boldsymbol{C} \cdot(s-1)$. Circled values are minimal for the given $\boldsymbol{C}$.

The obvious conclusion is that when organizational planning is inexpensive, more of it should be done. (For example, when $C=.1$, the project should be divided into $s=50$ subtasks of duration $U=2$ time units.) When planning is costly, unless the organizational cost can be distributed over several similar projects, planning time is harder to justify-one may as well suffer some of the effects of interruptions.

In closing, it seems appropriate to remind the reader that we have been engaging in an amusing flight of fancy. Estimation of project completion times and probabilities of interruption requires imaginative speculation. Hence calculations based on them lead to results still more imaginary. Like many flights of fancy our exploration is, however, enlightening. Effective scheduling of time remains pure art. Yet an excursion into the fancy of formulas and calculations can show how to extend the practice of that art.

\section{References}

[1] Morris H. DeGroot, Probability and Statistics, Addison-Wesley, Reading, MA, 1975.

[2] B. W. Lindgren and G. W. McElrath, Introduction to Probability and Statistics, 2nd ed., Macmillan, New York, 1966.

[3] Marcel F. Neuts, Probability, Allyn and Bacon, Boston, 1973.

[4] Herbert A. Simon, The Sciences of the Artificial, 2nd ed., MIT Press, Cambridge, MA, 1981, pp. $200-202$.

[5] George B. Thomas, Jr. and Ross L. Finney, Calculus and Analytic Geometry, 5th ed., Addison-Wesley, Reading, MA, 1979, Section 16-3. 